\title{
The softening of two-dimensional colloidal crystals
}

\author{
J Zanghellini, P Keim and H H von Grünberg \\ Institute of Chemistry, Karl-Franzens-University Graz, 8010 Graz, Austria
}

Received 28 September 2005

Published 28 October 2005

Online at stacks.iop.org/JPhysCM/17/S3579

\begin{abstract}
According to the Kosterlitz-Thouless-Halperin-Nelson-Young (KTHNY) theory a two-dimensional (2D) crystal melts when bound pairs of dislocations dissociate into single dislocations. The temperature at which dislocation pairs unbind can be identified by means of the Young's modulus $K$ passing through $16 \pi$. In a previous paper (von Grünberg et al 2004 Phys. Rev. Lett. 93 255703) we analysed video-microscopy data of $2 \mathrm{D}$ colloidal crystals, obtained elastic constants from normal-mode band-structures, and confirmed that the unbinding temperature is indeed close to the melting temperature. Processing the same data we now obtain elastic constants directly from an analysis in real space by computing relative mean-square displacements as a function of the lattice site separation. We have also carried out Monte Carlo simulations to check for the thermal softening of the colloidal crystal. Both studies confirm our previous results. We finally compare our results to the corresponding curves for a $2 \mathrm{D}$ electron solid on the surface of liquid helium.
\end{abstract}

(Some figures in this article are in colour only in the electronic version)

\section{Introduction}

In two-dimensional (2D) crystals melting may occur via two consecutive Kosterlitz-Thoulesstype [1] transitions. The first of these transitions takes the solid to a hexatic phase [2]. While in the 2D crystal the positional correlation function exhibits a power law decay, in the hexatic phase, like in the fluid phase, this correlation function decays exponentially. However, the correlation function of the orientational order parameter retains an algebraic decay in the hexatic phase. Only via a second Kosterlitz-Thouless-type transition does the system become an ordinary fluid where all correlation functions have an exponential decay. This two-stage scenario of 2D melting is described by Halperin, Nelson and Young [2, 3], and is also known as KTHNY theory (Kosterlitz, Thouless, Halperin, Nelson and Young). The two-stage KTHNY melting scenario describes only one way in which a 2D crystal could melt; others are possible. It is however clear by now that KTHNY melting takes place only in crystals with sufficient rotational stiffness, as has been clarified by a simple lattice defect model [4]. Detailed reviews of the extensive literature on this theory are available $[5,6]$. 
According to the KTHNY theory the translational symmetry of the crystal is destroyed by free dislocations. These dislocations stem from a dissociation of bound dislocation pairs (having a pair distance $R$ ) which are usually present in warm crystals. The dislocation unbinding temperature, i.e. the temperature where $\left\langle R^{2}\right\rangle \rightarrow \infty$, is reached when the Young's modulus

$$
K(T)=\frac{4 \mu(T) a_{0}^{2}}{k T} \frac{\lambda(T)+\mu(T)}{\lambda(T)+2 \mu(T)},
$$

passes through $16 \pi$ [6]. Here $\lambda$ and $\mu$ are the Lamé coefficients, and $a_{0}$ is the lattice constant of the $2 \mathrm{D}$ crystal. On the other hand, the real melting temperature $T_{\mathrm{m}}$ can be inferred from the behaviour of the correlation functions. So, it is clear that the KTHNY melting scenario can only be a correct description provided that indeed $K(T)$ passes through $16 \pi$ just at a temperature which from an analysis of the correlation functions is known to be the melting temperature. We should remark that this is only a necessary condition for a KTHNY melting, but not necessarily a sufficient one. Therefore, provided the KTHNY theory applies, the unbinding temperature must be equal to the melting temperature, that is

$$
\lim _{T \rightarrow T_{\mathrm{m}}^{-}} K(T)=16 \pi .
$$

We remark that the renormalized elastic constants are to be taken in (1). The renormalization of the constants takes account of the dislocation-induced softening of the crystal which ultimately causes melting. Differential equations to compute the renormalized elastic constants from the constants of the defect-free crystal can be found in [2].

In a recent paper [7], we have been able to verify (2) for a 2D colloidal system by analysing real-space video-microscopy data. Here we add two observations that are needed to complete the conclusions drawn in [7]. (i) Throughout [7] we have assumed elastic constants at $T=0$ for the defect-free crystal. However, in real crystals the constants soften not only due to defects but also due to phonon-phonon interactions (thermal softening [6]). By means of Monte Carlo (MC) simulation, we here check for the effect of thermal softening. (ii) In [7] real-space data are first transformed to $\boldsymbol{q}$-space, and the elastic constants are then obtained from an analysis of the $q \rightarrow 0$ behaviour of the elastic band-structure, following a method suggested in [8]. We now obtain elastic constants from a more direct analysis in real space which uses an information contained in the distance dependence of the mean-square relative displacement. We process the same data as in [7], and show that in real space they satisfy a relation similar to (2), too.

\section{Simulation: temperature dependence of the elastic constants}

The colloids used in [7] interact via a repulsive pair-potential, $u(r)$, which reads

$$
\beta u(r)=\frac{\Gamma}{(\sqrt{\pi \rho} r)^{3}} .
$$

Here, $r$ refers to the inter-particle distance, $\Gamma$ represents a dimensionless interaction amplitude and $\rho$ denotes the particle area density. The interaction potential, $u(r)$, is measured in units of $k T[\beta=1 /(k T)]$, with Boltzmann constant $k$ and temperature $T$. One may interpret $\Gamma$ as a reduced inverse temperature $T^{*}:=1 / \Gamma$. Moreover, $\Gamma$ is the only parameter controlling the phase behaviour of the system. For a system describable by equation (3), the melting temperature, $T_{\mathrm{m}}^{*}=1 / \Gamma_{\mathrm{m}}$, was found experimentally to be $1 / 60[10]$. For $\Gamma>60$, the colloidal system forms a hexagonal crystal.

Experimentally the potential in equation (3) can be realized by using spherical, superparamagnetic colloids confined to a water/air interface [9]. Applying a magnetic field $B$ 
Table 1. Conversion table.

\begin{tabular}{llll}
\hline Parameter & Conversion factor & Parameter & Conversion factor \\
\hline Length & $r \rightarrow r / a_{0}$ & Density & $\rho^{*}:=\rho a_{0}^{2}=2 / \sqrt{3}$ \\
Area & $A^{*}:=A / a_{0}^{2}$ & Temperature & $T^{*}:=1 / \Gamma$ \\
Energy & $E^{*}:=\beta E / \Gamma$ & Pressure & $p^{*}:=\beta p a_{0}^{2} / \Gamma$ \\
Lamé constants & $\lambda^{*}:=\beta \lambda a_{0}^{2} / \Gamma$ & & $\mu^{*}:=\beta \mu a_{0}^{2} / \Gamma$ \\
\hline
\end{tabular}

perpendicular to the water/air interface induces a linearly-dependent magnetic dipole moment leading to the interaction potential in (3), with $\Gamma=\left(\beta \mu_{0} / 4 \pi\right)(\chi B)^{2}(\sqrt{\pi \rho})^{3}$ and $\mu_{0}, \chi$ denoting the absolute magnetic permeability and the susceptibility. Thus by tuning $B$ one can easily control the interaction amplitude of the colloids. Such systems have been widely tested and are established as almost ideal 2D models [8, 7, 9-11]. We therefore refer to the aforementioned literature for experimental details. For all that follows we shall use reduced variables. Except for distances we will mark these reduced quantities with asterisks. Table 1 lists the corresponding conversion factors.

There are various ways to determine elastic constants by MC simulation [12]. For cubic crystals, in which particles interact with pairwise forces, Squire et al [13] have derived exact expressions for the three independent isothermal elastic constants, $C_{11}^{T *}, C_{12}^{T *}$, and $C_{44}^{T *}$. These quantities are connected to the Lamé constants via

$$
\lambda^{*}+2 \mu^{*}=C_{11}^{T *}-p^{*}, \quad \lambda^{*}=C_{12}^{T *}+p^{*}, \quad \mu^{*}=C_{44}^{T *}-p^{*} .
$$

Here $p^{*}=\rho^{*} T^{*}+\left\langle W^{*}\right\rangle / A^{*}$ denotes the pressure, which may be calculated via the internal virial, $\left\langle W^{*}\right\rangle, \rho^{*}$ presents the particle density, $T^{*}$ the temperature, and $A^{*}$ the sample area. Using the interaction potential given by equation (3) the reduced Lamé constants then read

$\mu^{*}=\frac{9 \Gamma}{\left(\pi \rho^{*}\right)^{3} A^{*}} \delta F\left[\Delta x_{i j} \Delta y_{i j}, \Delta x_{i j} \Delta y_{i j}, r_{i j}\right]+\frac{15}{\left(\pi \rho^{*}\right)^{3 / 2} A^{*}} G\left[\Delta x_{i j} \Delta y_{i j}, r_{i j}\right]-\frac{3 U^{*}}{2 A^{*}}$

$\lambda^{*}=\frac{9 \Gamma}{\left(\pi \rho^{*}\right)^{3} A^{*}} \delta F\left[\left(\Delta x_{i j}\right)^{2},\left(\Delta y_{i j}\right)^{2}, r_{i j}\right]+\frac{15}{\left(\pi \rho^{*}\right)^{3 / 2} A^{*}} G\left[\Delta x_{i j} \Delta y_{i j}, r_{i j}\right]+\rho^{*} T^{*}+\frac{3 U^{*}}{2 A^{*}}$

where $\Delta x_{i j}$ and $\Delta y_{i j}$ represent the $x$ and $y$ Cartesian coordinates of the inter-particle separation vector $\boldsymbol{r}_{i}-\boldsymbol{r}_{j}$ of modulus $r_{i j}$ and the reduced internal energy $U^{*}$ is given by $U^{*}=\left(\pi \rho^{*}\right)^{-3 / 2} \sum_{i, i<j} r_{i j}^{-3}$. In addition, we have defined the 'fluctuation function', $\delta F\left(\zeta_{i j}, \eta_{i j}, \theta_{i j}\right):=\left\langle\sum_{i, i<j} \zeta_{i j} / \theta_{i j}^{5}\right\rangle\left\langle\sum_{i, i<j} \eta_{i j} / \theta_{i j}^{5}\right\rangle-\left\langle\left(\sum_{i, i<j} \zeta_{i j} / \theta_{i j}^{5}\right)\left(\sum_{i, i<j} \eta_{i j} / \theta_{i j}^{5}\right)\right\rangle$ and a function $G\left(\eta_{i j}, \theta_{i j}\right):=\left\langle\sum_{i, i<j} \eta_{i j}^{2} / \theta_{i j}^{7}\right\rangle$. In the zero-temperature limit $\Gamma \rightarrow \infty \Rightarrow\{\delta F \rightarrow 0$, $\left.G \rightarrow(1 / 8) \sum_{i, i<j} \theta_{i j}^{-3}\right\}$ the Lamé constants $\lambda^{*}$ and $\mu^{*}$ converge toward 3.114 and 0.346 , respectively.

We evaluated equations (5) and (6) as a function of the inverse temperature $\Gamma$ using MC simulations, for a system of particles interacting according to equation (3). The results are depicted in figure 1. These simulations were performed using 625 particles in a periodically replicated rectangular unit cell, corresponding to a $25 \times 25$ super-cell of an unstrained lattice. This is a fairly small system which does not allow us to study transitions from solid to hexatic phases [14]. However, it may be considered appropriate as our analysis focuses on the temperature dependence of the elastic constants deep in the solid phase. On increasing the total number of particles up to 2000, the shear modulus, $\mu^{*}$, for instance, changes by less than $4 \%$. We have suppressed the long-range part of the potential for radii bigger than a cut-off radius $r_{\mathrm{c}}=9.74$. However, we have added the usual long-range correction terms [15] to correct for the truncation of the sums in $U^{*}, G$ and $\delta F$. The results are almost insensitive ( $<2 \%$ deviation) to the cut-off radius. We have started the calculation with $\Gamma=1000$ using 

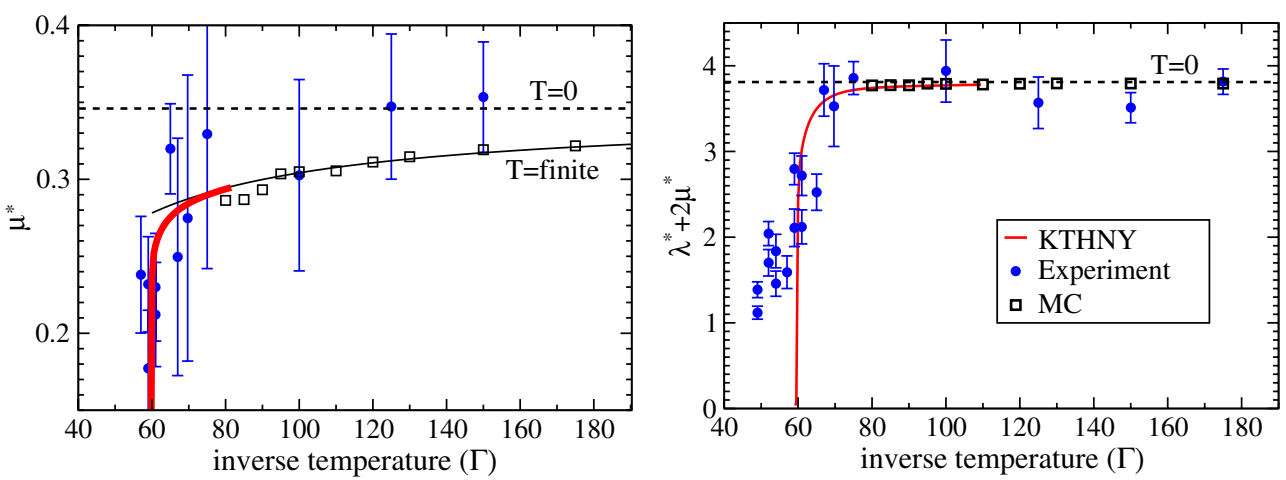

Figure 1. Monte Carlo evaluation of the elastic moduli given by equations (5) and (6) (empty squares) as a function of the inverse temperature $\Gamma$. In the left panel these data have been fitted and extrapolated to the inverse melting temperature $\Gamma_{\mathrm{m}}=60$ (thin solid line). Thick lines represent the elastic moduli as predicted by [2], dashed lines are the zero-temperature moduli. Full dots correspond to experimental data taken from [7].

an unstrained lattice as initial configuration, propagating it for typically 100000 time steps. At $\Gamma=1000$ we were able to reproduce the $T=0$ values of the elastic constants. Near the melting point, at $\Gamma_{\mathrm{m}}=60$, we have not been able to obtain converged results even for larger systems or considerably longer runs. The accessible $\Gamma$-range was $1000>\Gamma>75$ for the computation of $\mu^{*}$ and $\lambda^{*}$. These convergence difficulties near the transition are well-known and are thoroughly discussed in [16].

In figure 1 we compare the Lamé elastic constants predicted by the KTHNY theory (full thick lines) to experimental data of [7] (full dots) as well as to our MC simulations (empty squares). The MC data now reveal that for $\lambda^{*}+2 \mu^{*}$ the $T=0$ assumption is indeed a valid approximation for a broad range of $\Gamma$ values, down to values as low as $\Gamma=75$. $\mu^{*}$, on the other hand, shows a strong temperature effect; the $T=0$ approximation is reasonable only at much higher values of $\Gamma(\Gamma>500)$. The softening of the elastic constants at higher $\Gamma(\Gamma>85$, see below $)$ is not caused by topological defects, but is mainly due to higher anharmonic terms. From the known band-structure of this system [8], one could in principle calculate the thermal softening of the crystal [17]. More general considerations also show [17] that the transverse elastic constant should suffer much stronger from thermal softening than the longitudinal constant which is indeed what we here observe. We have fitted the simulation data of $\mu^{*} \Gamma$ to a straight line and obtained $\mu^{*} \Gamma=0.343(\Gamma-11.39)$ as the fit formula, which is plotted as solid thin line in the left panel of figure 1.

By means of this curve we can extrapolate the effect of thermal softening on $\mu^{*}$ down to the melting point at $\Gamma_{\mathrm{m}}=60$, leading to a value of $\mu=0.28$ at $\Gamma_{\mathrm{m}}$. This is the value that one would expect to find if the softening was produced exclusively by the phonon-phonon interaction in a perfect, i.e. defect-free, system. However, the experimental data points of the elastic constants at $\Gamma_{\mathrm{m}}=60$ are in fact much smaller. This extra softening is due to interacting dislocations, as predicted by the KTHNY theory. The KTHNY curve in figure 1 was obtained from integrating the renormalization-group equations in [2] using the $T=0$ value $\lambda^{*}=3.114$ for the longitudinal constant and the fit formula $\mu^{*} \Gamma=0.343 *(\Gamma-11.39)$ for the transversal constant to set up the boundary conditions at $\Gamma \rightarrow \infty$. Solution of these equations requires also a value for the dislocation core energy $E_{\mathrm{c}}$, a quantity which is hard to compute directly. We have therefore taken $E_{\mathrm{c}}$ to be a variable which was chosen such that the resulting elastic constants vanish at the known melting temperature of $\Gamma_{\mathrm{m}}=60$. We found $E_{\mathrm{c}}$ 


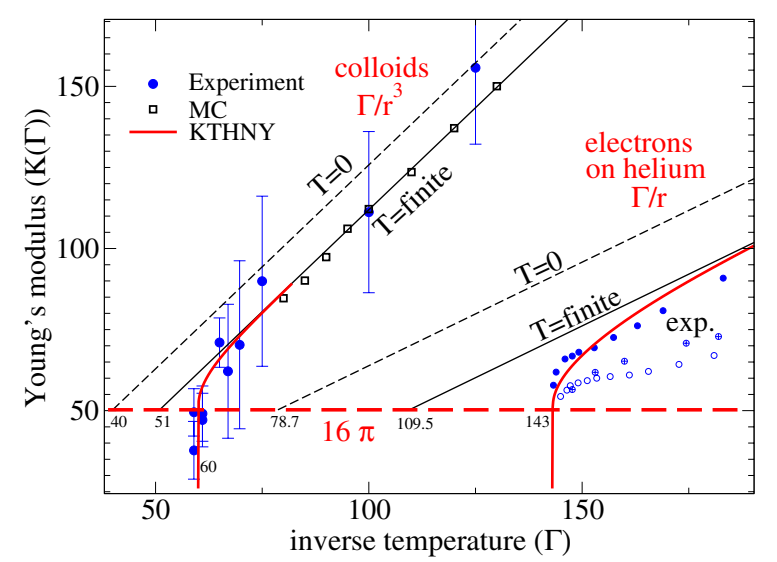

Figure 2. The Young's modulus as defined in equation (1) as a function of $\Gamma$ for our $2 \mathrm{D}$ colloidal crystal (pair interaction $\sim \Gamma / r^{3}$ ) and for a $2 \mathrm{D}$ electron crystal (interaction $\sim \Gamma / r$ ). In both systems, the Young's modulus has been approximated using elastic constants (i) from a $T=0$ calculation (dashed lines), (ii) from a finite temperature simulation (solid line) and (iii) from integrating the renormalization-group equations of the KTHNY theory (thick solid line). Experimental values are taken from [7] (colloids) and from [21] (electrons).

to be $5.7 k T$. The resulting KTHNY curve already reaches its limiting curves for $\Gamma \approx 85$, where the renormalization ceases to produce an effect. Thus, it is in the range $60<\Gamma<85$ where the dislocations are expected to soften the elastic constants, in addition to the thermal softening. And, indeed, in this regime the experimental data points seem to confirm the predictions of the KTHNY theory.

Having $\mu^{*}$ and $\lambda^{*}$ at hand we can easily compute the Young's modulus $K$, equation (1), plotted in figure 2 as a function of $\Gamma$. The simulation data are shown as empty squares, and are fitted to a straight line $(K(\Gamma)=1.258(\Gamma-11))$. This line is shifted relative to the $T=0$ approximation of the Young's modulus, $K(\Gamma)=1.258 \Gamma$ (dashed line), an expression which one obtains by inserting $\lambda^{*}=3.114$ and $\mu^{*}=0.346$ in (1). This shift results from the temperature dependence of $\mu^{*}$ which is the dominant constant in (1), and can be attributed to phonon-phonon interactions. Applying the Kosterlitz-Thouless criterion for melting, equation (2), one would predict the system to melt at $\Gamma=40$ relying on the $T=0$ approximation of $K$, and at $\Gamma=51$ if the thermal softening is taken into account. However, the crystal melts at $\Gamma_{\mathrm{m}}=60$, and the experimental data points closely follow the KTHNY curve, and cross $16 \pi$ very close to $\Gamma_{\mathrm{m}}=60$. Thus, the unbinding temperature is equal to the melting temperature. This is the main finding of [7]. However, in [7] we compared the experimental data points to a KTHNY curve converging to the $T=0$ curve of $K$, while now we have included the temperature dependence of $K$ by allowing the KTHNY curves to converge to $K(\Gamma)=1.258(\Gamma-11)$. The data points and the KTHNY curves still agree within the experimental error bars. The essential conclusion here is that judged on the basis of a thermally softened $K$ one would expect the Young's modulus to pass $16 \pi$ at $\Gamma=51$, while including the additional effect of the dislocations onto $K$ leads to a crossing of the $16 \pi$ line at a higher value of $\Gamma\left(\Gamma_{\mathrm{m}}=60\right)$. This difference shows that the temperature dependence of $K$ is not only produced by interacting phonons, but near $\Gamma_{\mathrm{m}}$ also by dislocations. This is important supplementary information completing the conclusions drawn in [7]. We should, however, remark that from these observations we can infer nothing regarding the order of the transition.

We next compare our results to the corresponding curves for a $2 \mathrm{D}$ electron solid on the surface of liquid helium, an experimental system that in the past has received much 
attention. Here, the interaction is $\beta u(r)=\Gamma /(\sqrt{\pi \rho} r)$, where $\Gamma=\sqrt{\pi \rho} \beta e^{2}$ ( $e$ is the electron charge, $\rho$ the area density). The $T=0$ values for the elastic constants are [19] $\lambda=\infty$ and $\mu=0.245065 e^{2} \rho^{3 / 2}$, so $\mu^{*}=0.15965$. Inserting these values in (1), one obtains $K(\Gamma)=0.6386 \Gamma$, plotted as a dashed line in figure 2 . This line crosses $16 \pi$ at $\Gamma=78.7$, an estimate for the melting temperature first given by Thouless [18]. Later Morf [20] determined the temperature dependence of the shear modulus of the $2 \mathrm{D}$ electron solid in a computer simulation and fitted his data points to $K(\Gamma)=0.6386(\Gamma-30.8)$, which in figure 2 is seen to cross $16 \pi$ at $\Gamma=109.5$. Morf then solved the renormalization relations in [2], taking this curve to define the boundary conditions plus an estimated value for $E_{\mathrm{c}}$, and was thus able to predict a melting temperature of $\Gamma_{\mathrm{m}}=128.2$. Figure 2 now shows the experimental data points for a $2 \mathrm{D}$ electron system on helium as obtained by Gallet et al [21] from a measurement of the coupled electron-substrate transverse sound mode for three different electron densities. All three data sets pass through $16 \pi$ at a common value of $\Gamma$ which is near to 143 , but unfortunately fail to fall onto a common curve as one would expect them to do. These data points are to be compared to the KTHNY curve (thick solid curve in figure 2) which we here computed following the same recipe as used for our colloidal system ( $T=$ finite values of $K$ at $\Gamma \rightarrow \infty$ and variation of $E_{\mathrm{c}}$ to reproduce a given $\Gamma_{\mathrm{m}}$; resulting in $E_{\mathrm{c}}=5.4 k T$ ). At least one experimental data set is now in good agreement with the curve predicted by the KTHNY theory. It is interesting to compare the sequence of melting points predicted by (2) on the basis of different approximations for $K$ in the electron system (interaction $\sim \Gamma / r$ ) and the colloidal system (interaction $\sim \Gamma / r^{3}$ ). In the electron system we have for $\Gamma_{\mathrm{m}}$ the values $78.7 \rightarrow 109.5 \rightarrow 143$, while in the colloid system we find $40 \rightarrow 51 \rightarrow 60$, estimating the Young's modulus first by the $T=0$, then by the $T=$ finite approximation and finally through its renormalized values. The shrinking of the range covered by these three melting temperatures in going from a $\Gamma / r$ to a $\Gamma / r^{3}$ pair potential seem to suggest that a more short-ranged potential such as $\Gamma / r^{5}$ would lead to an even smaller interval for these three temperatures, making it obviously increasingly difficult to distinguish between a defect-mediated and a phonon-mediated melting.

\section{Experiment: temperature dependence of the relative mean-square displacement}

We now again process the video-microscopy data of [7]. These data are based on the sampling of approximately 2000 particle trajectories (taken from a pool of roughly $10^{5}$ particles) typically recorded for $2 \mathrm{~h}$ leading to about 3600 configurations. It is the extremely long equilibration time plus the huge system size that sets the experimental real-space data most prominently apart from data obtained from ordinary simulation. In what follows we again check the experimental elastic constants obtained in [7] and plotted in figure 1, but now compute the elastic constants directly from real-space data without first transforming to the reciprocal space. We investigate the dependence of the mean-square relative displacement

$$
\left\langle W_{i j}\left(\left|\boldsymbol{R}_{i}-\boldsymbol{R}_{j}\right|\right)\right\rangle=\left\langle\left[\boldsymbol{u}\left(\boldsymbol{R}_{i}\right)-\boldsymbol{u}\left(\boldsymbol{R}_{j}\right)\right]^{2}\right\rangle
$$

with $\boldsymbol{u}\left(\boldsymbol{R}_{i}\right)=\boldsymbol{r}_{i}-\boldsymbol{R}_{i}$ and the lattice site denoted by $\boldsymbol{R}_{i}$. Experimentally we associate the average of all configurations, $\left\langle\boldsymbol{r}_{i}\right\rangle_{t_{\exp }}$, with a lattice site. One important result of the KTHNY theory is that for large lattice-site separation $\left|\boldsymbol{R}_{i}-\boldsymbol{R}_{j}\right|$ the mean-square relative displacement asymptotically approaches [2]

$$
\left\langle W_{i j}\left(\left|\boldsymbol{R}_{i}-\boldsymbol{R}_{j}\right|\right)\right\rangle \rightarrow \eta(\Gamma) \ln \left(\left|\boldsymbol{R}_{i}-\boldsymbol{R}_{j}\right| / L\right)+C(\Gamma),
$$

with slope

$$
\eta=\frac{\lambda^{*}+3 \mu^{*}}{\pi \Gamma \mu^{*}\left(\lambda^{*}+2 \mu^{*}\right)}
$$



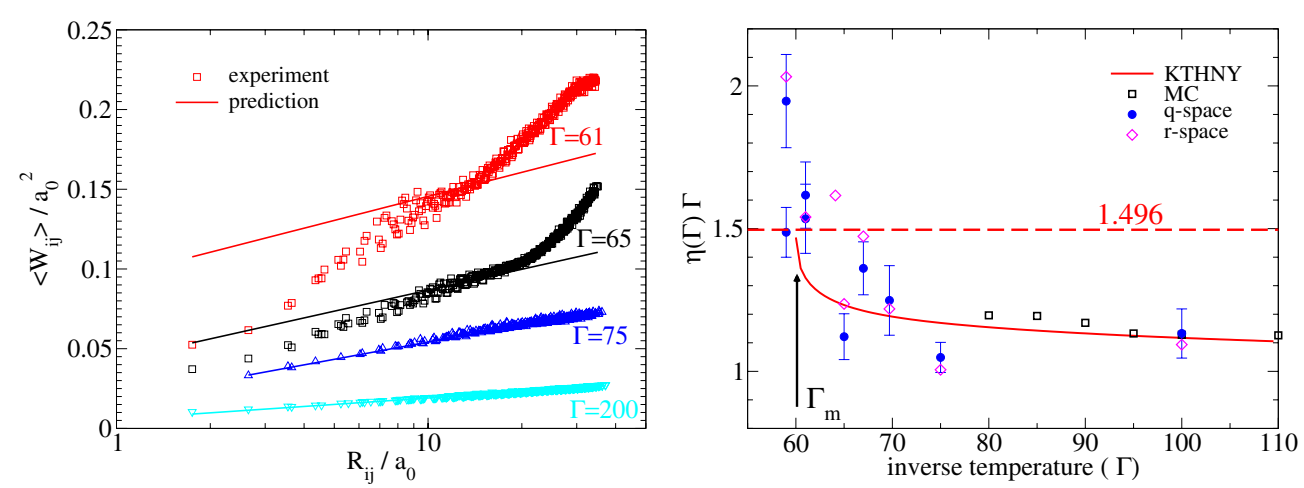

Figure 3. Left panel: thermal average of the mean-square relative displacement, $\left\langle W_{i j}\right\rangle$, of a $2 \mathrm{D}$ colloidal crystal as a function of the lattice site separation, $R_{i j}=\left|\boldsymbol{R}_{i}-\boldsymbol{R}_{j}\right|$, for various inverse temperatures, $\Gamma$. The thick lines represent curves according to (8) with an $\eta$ as predicted by [2]. Right panel: experimental, simulational and theoretical values for $\eta \Gamma$ as a function of the inverse temperature. This plot is the pendant to figure 2: like $K \rightarrow 16 \pi, \eta$ must pass 1.496 at the melting transition if the crystals melts according to the KTHNY theory.

and some irrelevant constants $L$ and $C$. At $T_{\mathrm{m}}, \eta$ becomes an universal function of the 2D Poisson's ratio $\sigma=\lambda^{*} /\left(\lambda^{*}+2 \mu^{*}\right)$, and can be written, using (2), as [2]

$$
\lim _{T \rightarrow T_{\mathrm{m}}^{-}} \eta=\frac{1}{16 \pi^{2}} \lim _{T \rightarrow T_{\mathrm{m}}^{-}}(1+\sigma)(3-\sigma)
$$

which approaches $1 /\left(4 \pi^{2}\right)$ for $\sigma=1$ and $0.985 /\left(4 \pi^{2}\right)$ for $\sigma=0.751$, the value we find in our case at hand taking $\lambda^{*}$ and $\mu^{*}$ at $\Gamma_{\mathrm{m}}=60$ from figure 1 . Accordingly, $\eta \Gamma$ goes to 1.496 at $\Gamma_{\mathrm{m}}=60$. Equation (10) may be considered as the real-space counterpart of equation (2), and is here checked with values of $\eta$ obtained from exploiting (8).

The left panel of figure 3 is a logarithmic plot of $\left\langle W_{i j}\left(\left|\boldsymbol{R}_{i}-\boldsymbol{R}_{j}\right|\right)\right\rangle$ as a function of $\left|\boldsymbol{R}_{i}-\boldsymbol{R}_{j}\right|$ evaluated from experimental data (points) for four different temperatures. At low temperatures, we indeed obtain straight lines from which we can easily determine $\eta$. For higher temperatures $(\Gamma=65)$, slightly above $T_{\mathrm{m}}^{*}$, we notice three different regimes. At very large distance, the curve shows an up-bending which still depends on the number of configurations processed and is probably due to insufficient sampling. $\left\langle W_{i j}\left(\left|\boldsymbol{R}_{i}-\boldsymbol{R}_{j}\right|\right)\right\rangle$ at small distances is also not useful, as (8) is valid only for large values of $R_{i j}$. We have therefore tried to identify the intermediate regime ${ }^{1}$, and determined the slope only from these data points. The right panel in figure 3 shows $\eta \Gamma$, thus determined, as a function of $\Gamma$ (diamonds). These data are compared to values of $\eta \Gamma$ obtained from (9) with elastic constants determined (i) experimentally in [7] in $q$-space, and (ii) following the renormalization procedure of [2] (solid lines in figure 1). The good agreement between the experimental data points derived in $\boldsymbol{q}$ - and $\boldsymbol{r}$-space validates once more the method we applied in [7] to derive elastic constants. We also observe that $\eta$ crosses the value predicted by (10) very close to the melting temperature $\Gamma_{\mathrm{m}}=60$, just like $K$ passes through $16 \pi$ at $\Gamma_{\mathrm{m}}$, pointing again to a KTHNY melting scenario. We should finally remark that determining elastic constants via (8) is not always unproblematic near $T_{\mathrm{m}}$, where it can become difficult to identify the relevant intermediate regime. To demonstrate that also

\footnotetext{
1 We first identified the largest distance $R_{i j}^{\max }$ below which the data do not suffer from a dependence on system-size or total measurement time. The interval $\left[0, R_{i j}^{\max }\right]$ was then separated into two adjacent intervals $\left[0, R_{i j}^{x}\right]$ and $\left[R_{i j}^{x}, R_{i j}^{\max }\right]$ by setting $R_{i j}^{x}$, and independent fits $\sim \log R_{i j}$ were made in both intervals. The final $R_{i j}^{x}$ were finally chosen such as to optimize the fits in both intervals. $\left[R_{i j}^{x}, R_{i j}^{\max }\right]$ is then the intermediate regime.
} 
the temperature dependence of $\eta$ is well estimated by KTHNY theory, it is therefore probably better to compare the experimental $\left\langle W_{i j}\right\rangle$ to curves following equation (8) with an $\eta$ estimated from the KTHNY theory, as illustrated in the left panel of figure 3.

\section{References}

[1] Kosterlitz J and Thouless D 1973 J. Phys. C: Solid State Phys. 61181

[2] Nelson D R and Halperin B I 1979 Phys. Rev. B 192457

[3] Young A 1979 Phys. Rev. B 191855

[4] Kleinert H 1988 Phys. Lett. A 130443

Kleinert H 1989 Phys. Lett. A 136468

Janke W and Kleinert H 1988 Phys. Rev. Lett. 612344

[5] Strandburg K J 1988 Rev. Mod. Phys. 60161

Glaser M and Clark N 1993 Adv. Chem. Phys. 83543

Bruch L W, Cole M W and Zaremba E 1997 Physikcal Adsorption, Forces and Phenomena (Oxford: Clarendon)

[6] Kleinert H 1989 Gauge Fields in Condensed Matter vol II (Singapore: World Scientific)

[7] von Grünberg H H, Keim P, Zahn K and Maret G 2004 Phys. Rev. Lett. 93255703

[8] Keim P, Maret G, Herz U and von Grünberg H H 2004 Phys. Rev. Lett. 92215504

[9] Zahn K, Mendez-Alcaraz J M and Maret G 1997 Phys. Rev. Lett. 79175

[10] Zahn K, Lenke R and Maret G 1999 Phys. Rev. Lett. 822721

Zahn K and Maret G 2000 Phys. Rev. Lett. 853656

[11] Zahn K, Wille A, Maret G, Sengupta S and Nielaba P 2003 Phys. Rev. Lett. 90155506

Zahn K, Maret G, Ruß C and von Grünberg H H 2003 Phys. Rev. Lett. 91115502

[12] Frenkel D and Smith B 2002 Understanding Molecular Simulation (San Diego, CA: Academic) see appendix $\mathrm{C} 4$

[13] Squire D, Holt A and Hoover W 1969 Physica 42388

[14] Chen K, Kaplan T and Mostoller M 1995 Phys. Rev. Lett. 744019

[15] Allen M and Tildesley D 2003 Computer Simulations of Liquids (New York: Oxford University Press)

[16] Naidoo K and Schnitker J 1994 J. Chem. Phys. 1003114

[17] Kleinert H 1989 Gauge Fields in Condensed Matter vol II (Singapore: World Scientific) chapter 7.7

[18] Thouless D J 1978 J. Phys. C: Solid State Phys. 11 L189

[19] Bonsall L and Maradudin A A 1977 Phys. Rev. B 151959

[20] Morf R H 1979 Phys. Rev. Lett. 43931

[21] Gallet F, Deville G, Valdes A and Williams F I B 1982 Phys. Rev. Lett. 49212 